\title{
WELL TEST ANALYSIS AND INTERPRETATION: THE USE OF ARTIFICIAL NEURAL NETWORK
}

\author{
Samuel Lucky Arubi, Bibobra Ikporo, Sunday Igbani, Ann Obuebute \\ Department of Petroleum Engineering, Niger Delta University, Wilberforce Island, Bayelsa, Nigeria. \\ Sylvester Okotie \\ Department of Petroleum and Natural Gas Engineering, Federal University of Petroleum Resources, Effurun, \\ Delta, Nigeria.
}

\begin{abstract}
Throughout the life of a field, the only way to make contact with the reservoir is through the wellbore by making indirect data measurements. These data acquired during the measurement period is analyzed and interpreted in other to have a better understanding of the reservoir characteristics. There are several methods of estimating reservoir properties such as core analysis and well logging which are only able to obtain properties at a local point. But, well testing present an average property of the whole reservoir as the reservoir responds to the perturbation caused during the test period. This study developed an artificial neural network algorithm that was trained to automatically identify four different reservoir models (the homogenous infinite acting radial flow with wellbore storage reservoir, homogenous reservoir with a finite conductivity fracture, homogenous reservoir with an infinite conductivity fracture and a double porosity reservoir with no wellbore storage effect) and estimate the model parameters (permeability, skin factor, reservoir radius, flowing wellbore pressure and/or the length of the conductivity fracture). The algorithm was constrained to four models because of the unavailability of well test data that represent the other plethora of reservoir models. The accuracy of the algorithm on reservoir model recognition was $99.5 \%$ and also, the reservoir model parameter estimation had an index of fitness of 1 for the four reservoirs and the mean squared error of $2.62653 \mathrm{e}-10$, $3.29122 \mathrm{e}-8,1.05805 \mathrm{e}-5$ and $2.19763 \mathrm{e}-6$ respectively. It is then concluded that an artificial neural network is a good tool for well test analysis and interpretation. And that it saves both the time and energy of the interpreter when compared to convention methods thereby providing enough time for proper engineering judgment.
\end{abstract}

Keywords - Well test, well test analysis, well test interpretation, artificial Intelligence; artificial neural network.

\section{INTRODUCTION}

Over the years, well test analysis has been used to assess and ascertain wellbore conditions and obtain reservoir parameters. Throughout the life of a field, the only way to make contact with the reservoir is through the wellbore by making indirect data measurements. These data acquired during the measurement period is analyzed and interpreted in other to have a better understanding of the reservoir characteristics.

The extent, quality and what is expected from a well test analysis and interpretation are limited to the state-of-the-art available both in data acquisition and data analysis [1]. He also added that as data acquisition improves and better interpretation methods are developed, more and more useful information can be extracted from a well test data.

Aforetime, the traditional well test has been timeconsuming and costly to perform, as production has to be stopped (loss of production time) for buildup tests and tools to be lowered into the well each time a well-test should be performed, however, the increased utilization of permanent gauges for continuous measurements has made the task of obtaining data for well-test analysis nearly straightforward such that well-test can now be done continuously with measured pressure and rate signals. Furthermore, the introduction of computers made the processing of the measured pressure and rate signal easier and well-test analysis was both improved and accelerated. Due to this continuous measurement of the pressure and rate signals, additional information about the reservoir and the wellbore can be derived in a more cost-effective manner and frequent way than it was before.

Well-test analysis and interpretation methods have evolved and been developed overtime. The analysis methods started from the straight-line method to the pressure type curve analysis to the type curve with independent variables and to the derivative plots and most recently the deconvolution algorithm [1]. Before the late 1970s, all well-test analysis methods were done by hand with the use of paper, pencils, and graphs. The application of computerized algorithms to the 


\section{International Journal of Engineering Applied Sciences and Technology, 2020 Vol. 4, Issue 11, ISSN No. 2455-2143, Pages 438-446 \\ Published Online March 2020 in IJEAST (http://www.ijeast.com)}

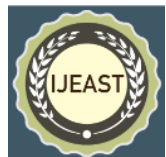

analysis and interpretation process began in the late 1970s which made the process easier and more effective for the interpreter [1]. According to Horne (1994) [2], the expansion in interpretation capability over the years aroused mainly from the development of computer-aided techniques. Each of the established methods was developed in other to overcome the limitations of the previous methods developed before it.

There are two main objectives when analyzing and interpreting well-test data. These objectives are to diagnose the underlying conceptual reservoir model and to estimate the model-related parameters [3,4,5,6]. The non-uniqueness problem, however, brings about the confusion in selecting the correct reservoir model using conventional approaches $[1,6]$. To meet these objectives stated above, different technologies and algorithms have been implemented. [6], [7], [8], [9], and [10] all used a different form of artificial neural network (ANN) to achieve the above-stated well-test objectives.

However, many approaches have been developed to investigate well-testing reservoir model identification using artificial intelligence (AI) techniques but only a few techniques have been developed to estimate the model-related parameters [6]. This paper aimed at developing an artificial neural network algorithm that analyzes and interprets a well test data automatically.

\subsection{Reservoir Model Identification}

In the words of Horne (1994) [2] I quote, 'Because the underlying principle of well-test interpretation is to match a mathematical model to an observed reservoir response, it is necessary first to decide which mathematical model, out of the plethora available is most appropriate to the actual reservoir'. Also, a correct well-test interpretation model is the basement of well-test analysis which is identified by the feature of pressure derivative curves [11]. In addition, correct model identification is an essential component of well-test interpretation, if the model is wrong, the resulting parameter estimates are useless [6].

Therefore, in other to prevent a futile job of interpreting a pressure transient data based on the wrong model, different researchers have developed and proposed different ways of identifying the reservoir models.

[12] proposed a graphical analysis method using the pressure derivative plot and it has become a standard procedure for reservoir model recognition. The development of computer-aided interpretation algorithms has increased over the years. However, [13] stated that computers have mainly been used to solve the easiest part of the interpretation process. And that the most difficult parts namely, reservoir model identification and validation of the analysis have received little attention. He also added that most computer approaches only aim at reproducing or facilitating the manual process and are not capable of bringing much improvement in analysis capability. And that some computer approaches, on the other hand, involve the use of black-box regression packages coupled to a series of interpretation models and can be dangerously misleading.
In an attempt to automate the well-test model recognition process, [7] gave the first attempt using artificial intelligence techniques. They employed syntactic pattern recognition and rule-based models from the pressure derivative plots.

[15] implemented feature extraction in a hyper-stack that used the human operator to describe features of the data. Also, [7] used a symbolic representation approach achieving feature extraction by developing a sketch of the data.

The neural network approach has the advantage of being insensitive to noise and can recognize the reservoir model despite intervening non-idealities in the data. Anyways, the disadvantage of the neural network approach is that it has no vision of the characteristics of the data and therefore allows no further reasoning as to which part of the data is associated with specific reservoir flow regimes. However, [16] described a hybrid approach in other to automated the model recognition process and overcome the disadvantages of conventional methods.

Furthermore, [8] used a modular neural network to identify different model classes. Also, [9] proposed a similar method that uses multiple neural networks with each network representing a single conceptual reservoir model. This is to overcome the disadvantages of using a single comprehensive neural network for covering all possible reservoir models.

However, [2] stated that various reservoir and well configurations have easily recognizable characteristics on a pressure derivative plot and that this one-plot solution significantly expanded the possibilities for automation of model recognition.

[3] proposed the use of a higher-order neural networks (HONN) instead of conventional multilayer perceptron (MLP) networks in identifying the well-test interpretation model regarding both scale and translation invariance of the well-test model to field data.

Similarly, [17] in their work developed a method based on an ANN that uses Kohonen's self-organizing feature (SOF) mapping technique to identify well-test interpretation models.

Also, [18] suggested the use of Hough transform (HT) as a unique technique for the extraction of the basic shape and motion analysis in noisy images, combined with the backpropagation neural network to improve the well-test model identification. Furthermore, [11] proposed an ANN approach to automate the process of type curve matching and move the tested and move the tested curves to their sample positions. Unlike the previous approaches that used data point series as input vectors to train ANNs, the binary vectors of theory curves created by transferring the actual derivative curves into binary numbers were used as training samples to train the ANN.

[6] applied different ANN methodologies including MLP, probabilistic neural network and generalized regression neural network to identify the well-test interpretation model and 


\section{International Journal of Engineering Applied Sciences and Technology, 2020 \\ Vol. 4, Issue 11, ISSN No. 2455-2143, Pages 438-446 \\ Published Online March 2020 in IJEAST (http://www.ijeast.com)}

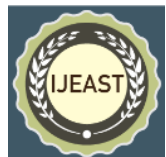

estimate the model-related variables from the pressure derivative curves.

Finally, identifying the conceptual or underlying reservoir models isn't enough but also the reservoir and wellbore parameters associated with the identified model must be estimated as accurately as possible.

\subsection{Reservoir Model Parameter Estimation}

It could be said that the major aim of carrying out well testing is to obtain reservoir and wellbore parameters. These parameters, however, are attributed to the conceptual reservoir model and can be estimated to a high degree of accuracy if the accurate reservoir model has been chosen. According to [19], the reservoir parameter estimation problem has many issues to be addressed and these problems are parameterization of the system resolution and uncertainty tradeoff, data integration, inversion procedure and preservation of geological structures. However, he added that many works have been done on these areas but the problems are still far from being eliminated.

Conventionally, reservoir parameters estimation algorithm is done using gradient-based optimization algorithms such as the quasi-newton [20], the Levenberg-Marquardt [21, 22], the steepest descent [23, 24], the conjugate gradient [25], and Gauss-Newton [26,27] methods.

[18] proposed the use of a recursive estimation method which accurately estimates the parameters even amid sparse available data. This recursive method can also ascertain the quality of the estimated parameters.

Furthermore, [29] instead of the conventional solutions in the time domain, explored the alternative of transforming the data into the Laplace domain and then fitted the model in the Laplace domain.

In addition, [30] presented a robust and efficient leastsquare algorithm for parameter estimation in well-test interpretation. The algorithm is a Levenberg-Marquardt with a trust region approach for global convergence along with restriction in the unknown parameters.

[31] also, obtained reservoir characteristics with a well-test model equipped with modified Levenberg-Marquardt methods. Likewise, [32] proposed a robust way of achieving a well test interpretation by combining the sequential predictive probability method with an artificial neural network approach. The sequential predictive probability method considers all possible reservoir models and determines the candidate model(s) that best predicts the well responses.

[19] proposed a new multi-resolution approach for the reservoir parameter estimation. They developed a model that integrated the wavelet analysis and nonlinear regression algorithm. The wavelet analysis is used to describe the distribution of sensitivity coefficients. This method is both reliable and efficient.

Furthermore, [10] proposed an ANN approach to estimate different model parameters for the faulted reservoir. The network development begins with a single architecture and a few inputs and output features. The levels of complexity of the system are heuristically and gradually increased as more model parameters tend to be predicted by the network.

[33] postulated a new methodology for the automated parameter estimation from well test data based on the typecurve matching using the signal theory. It could be noted that the presences of noise in the well test data set does not affect the solution from this procedure and that it is faster compared to the conventional techniques.

Similarly, [34] estimated reservoir (permeability) and wellbore (skin factor) parameter using a statistical approach to checkmate the quantitative interpretation of the derivative method. Also, [35] presented and compared three different grid-based inversion methods for the estimation of formation parameters and spatial geological feature identification based on pressure transient test data from multiple well locations. The first and the second methods employ efficient ad joint schemes to determine the gradient of the objective functions resulting in the most likely set of reservoir parameters and an ensemble of updated realization of parameters. The second method is based on the Langevin equation, while the third method uses the ensemble Kalman filtering for data assimilation, in which the outcome is an ensemble of parameter realization.

In addition, [36] presented the use of local-global optimization (particle swarm optimization) method that generates multiple realizations of reservoir parameters at a coarse-scale. The method involves the use of a local search optimization algorithm to parameterize the model space at a coarse-scale followed by a stochastic search for better estimation in the vicinity of the local estimates. Due to the problem of the non-uniqueness of solutions to the inverse problems of which well-test analysis and interpretation are, regularization has been used to reduce the effects of nonuniqueness [36].

Finally, it is observed that different algorithms and procedures have been developed to estimate the reservoir and wellbore parameters over the years.

\section{Method/Methodology}

Because the underlying principle of well-test interpretation is to match a mathematical model to an observed reservoir response (model parameter estimation) it is necessary first to decide which mathematical model out of the many available is most appropriate to the actual reservoir (model recognition).

Over the years, different methods such as type-curve matching, pressure derivative plots, etc. have been used to identify different reservoir models. In this study, an Artificial Neural Network would be used to achieve this purpose. The network would be trained on well test data already analyzed by well test experts. Due to the absence of sufficient well test data, the algorithm was trained to recognize only four reservoir types. These are the homogenous infinite acting radial flow 


\section{International Journal of Engineering Applied Sciences and Technology, 2020 \\ Vol. 4, Issue 11, ISSN No. 2455-2143, Pages 438-446 \\ Published Online March 2020 in IJEAST (http://www.ijeast.com)}

with wellbore storage reservoir, homogenous reservoir with a finite conductivity fracture, homogenous reservoir with an infinite conductivity fracture and a double porosity reservoir with no wellbore storage effect.

\subsection{Reservoir Model Recognition Artificial Neural Network Architecture (ANNA)}

A two-layered feed-forward network with a tanh-sigmoid activated hidden neuron and a softmax activated output neurons are used for model recognition in this paper. The network was trained with a scaled conjugate gradient backpropagated algorithm. Below is shown in figure 1 is the proposed neural network architecture;

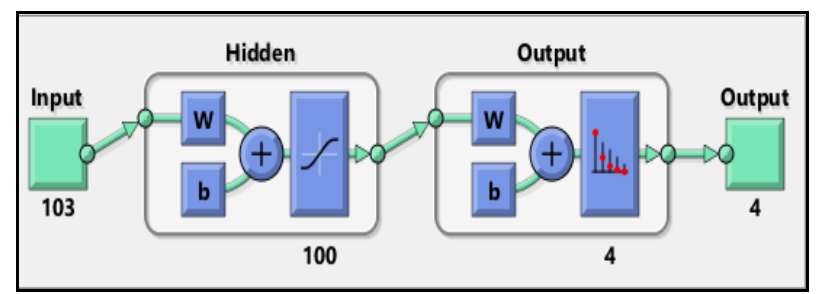

Figure 1: Model Recognition Artificial Neural Network Architecture

The input layer accepts well test (pressure and time) data, the hidden layer is made up of 100 neurons while the output layer is made up of 4 neurons which represent the 4 different reservoir types covered in this paper.

\subsubsection{Tanh-Sigmoid Activation (Transfer) Function}

The tanh-sigmoid activation function takes an input into a neuron that has a value between plus and minus infinity and then squashes it to output a value between the range of negative one to a positive one (-1 to 1$)$. Mathematically, the tanhsigmoid activation function is represented as;

$$
\begin{aligned}
\text { let } z & =\Theta^{T^{T}} x \\
g(z) & =\frac{2}{1+\theta^{-n Z}}-1
\end{aligned}
$$

Where;

$\mathrm{Z}$ is the vector product of the neuron weight transposed $\left(\Theta^{T}\right)$ and the input value (x).

$g(z)$ is the tanh-sigmoid transfer function.

This transfer function is commonly in backpropagation networks, in part because it's differentiable. Figure 2 below illustrates the tanh-sigmoid function;

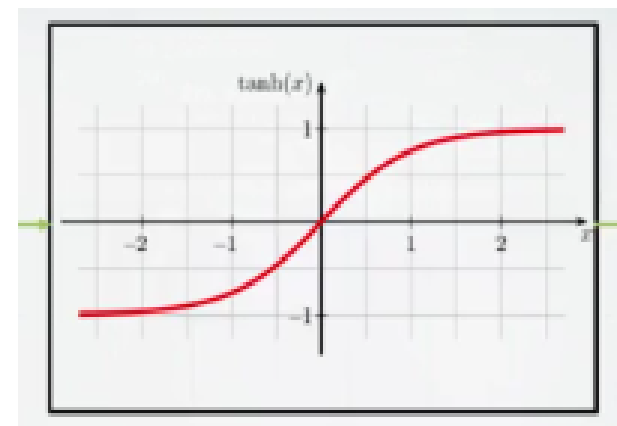

Figure 2:Graphic representation of the tanh-sigmoid transfer function

\subsubsection{Softmax Activation (Transfer) Function}

This function takes the input to the neuron (in this case output from the tanh-sigmoid hidden layer) and turns it into probabilities that sum to one. Softmax activation function outputs a vector that represents the probability distribution of a list of potential outcomes.

Mathematically, the softmax activation function is represented as

$$
h(z)_{i}=\frac{e^{z_{i}}}{\sum_{i=1}^{k} e^{z_{i}}}
$$

Where $\mathrm{k}$ is the total number of the potential output.

The softmax function is represented graphically in figure 3 below.

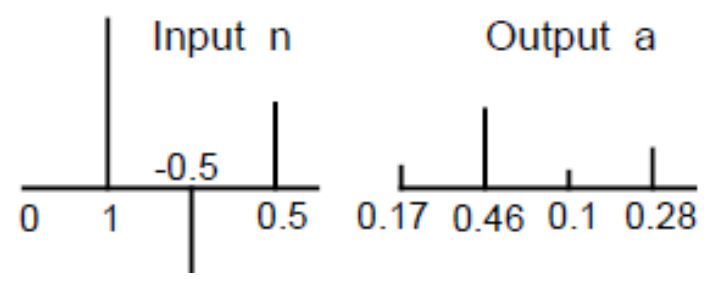

Figure 3: Graphical representation of the softmax activation function

\subsubsection{Cost (Error) Function}

The cost (error) function is the function used to minimize the error between the output (result by the network) and the target (actual result). Because pattern recognition is a logistic regression problem that does with discrete values of true or false ( 1 or 0$)$ the Cross-Entropy cost function was used to train the network. The Cross-Entropy function is given below;

$J(w)=-\frac{1}{m} \sum_{i=1}^{m}\left[y_{i} \log \hat{y}_{i}+\left(1-y_{i}\right) \log \left(1-\hat{y}_{i}\right)\right.$

Where $\mathrm{J}(\mathrm{w})$ is the cost function,

$\mathrm{m}$ is the number of training examples,

$y_{i}$ is the target vector of the training example $\mathrm{i}$,

$\widehat{y}_{i}$ is the output of the network for the training example $i$. 


\section{International Journal of Engineering Applied Sciences and Technology, 2020 \\ Vol. 4, Issue 11, ISSN No. 2455-2143, Pages 438-446 \\ Published Online March 2020 in IJEAST (http://www.ijeast.com)}

\subsubsection{Model Recognition Training Procedure}

The procedures with which the network was trained can be summarized in the following steps;

i. Import or read the training data (time and pressure data of the well test) from excel data sheet to MATLAB as variables.

ii. $\quad$ Preprocess the input data to prevent outliers

iii. Indicate the number of input and output neurons in this case hundred (100 and 4 respectively).

iv. Divide the input data randomly into three parts for training, validating and testing the algorithm (in this case the division was made in the ratio of $7: 2: 1)$ to prevent overfitting.

v. Indicate the transfer functions for the input and output neurons (in this case the tanh-sigmoid and softmax function respectively).

vi. Select the cost or error (in this case the CrossEntropy) function to be minimized.

vii. Choose the training algorithm, in this case, the scaled conjugate gradient backpropagation.

Train the network to meet the goal (until the cost function is minimized and the variables converge).

\subsection{Reservoir Model Parameter Estimation ANNA}

In other to estimate the parameter of the models that the recognition algorithm deemed fit to best represent the reservoir, a two-layered feed-forward network with a tanh-sigmoid activated hidden neuron and linear activated output neurons are used for parameter estimation. The parameters estimated by the model are reservoir permeability, skin factor, and reservoir radius of investigation, flowing bottom-hole pressure and the fracture length of fractured reservoirs. The network was trained with a Levenberg-Marquardt backpropagation algorithm. Figure 4 below, illustrates the proposed neural network architecture;

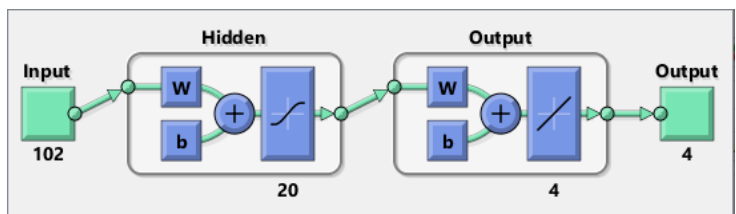

Figure 4: Model Parameter Estimation ANNA

\subsubsection{Linear Transfer Function}

This function takes the input to the neuron (in this case output from the tanh-sigmoid hidden layer), and turns it into a continuous number. Linear activation function calculates the neuron's output by simply returning the value passed to it. Mathematically, the linear activation function is represented as;

$$
\mathrm{a}=\operatorname{purelin}(\mathrm{n})=\operatorname{purelin}(\mathrm{Wp}+\mathrm{b})=\mathrm{Wp}+\mathrm{b}
$$

Where $\mathrm{W}$ is the weight vector, $\mathrm{p}$ is input value and $\mathrm{b}$ is the bias term. Figure 5 below illustrates the linear transfer function;

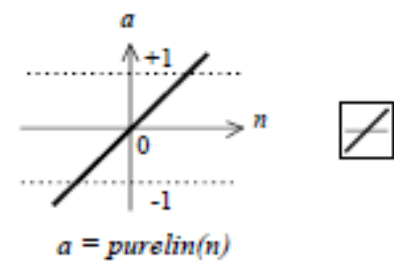

Figure 5: Graphical representation of the linear transfer function

\subsubsection{Error Function}

The cost (error) function is the function used to minimize the error between the output (result by the network) and the target (reservoir response). Because parameter estimation is a multiple regression analysis problem that output continuous values Mean Squared Error cost function was used to train the network. The Mean Squared Error cost function is given below;

$$
I=\frac{1}{m} \sum_{i=1}^{m}(P(i)-y(i))^{2}
$$

Where $\mathrm{P}$ is the model output of the training example $\mathrm{i}, \mathrm{y}=$ reservoir response of the training example $\mathrm{i}$.

Fortunately, the mean squared error performance index for the linear network is a quadratic function. Thus, the performance index will either have one global minimum, a week minimum or no minimum, depending on the characteristics of the input vectors. Specifically, the characteristics of the input vectors determine whether or not a unique solution exists.

\subsubsection{Model Parameter Estimation Training Procedure}

The procedures with which the network was trained can be summarized in the following steps;

i. Import or read the training data (time and pressure data of the well test) from excel data sheet to MATLAB as variables.

ii. Preprocess the input data to prevent outliers

iii. Indicate the number of input and output neurons (number of unknown reservoir parameters) in this case hundred (20 and 5 respectively).

iv. Divide the input data randomly into three parts for training, validating and testing of the algorithm (in this case the division was made in the ratio of $7: 1.5: 1.5)$ to prevent overfitting.

v. Indicate the transfer functions for the input and output neurons (in this case the tanh-sigmoid and linear function respectively).

vi. Select the cost or error (in this case the Mean Squared Error) function to be minimized.

vii. Choose the training algorithm, in this case, the Levenberg-Marquardt backpropagation.

viii. Train the network to meet the goal (until the cost function is minimized and the variables converge). 


\section{International Journal of Engineering Applied Sciences and Technology, 2020 \\ Vol. 4, Issue 11, ISSN No. 2455-2143, Pages 438-446 \\ Published Online March 2020 in IJEAST (http://www.ijeast.com)}

ix.

These steps i to viii are repeated for each reservoir type.

\subsection{Automation Process}

The model recognition and the parameter estimation algorithm would be integrated to form an automated system. The model recognition model first diagnoses and recognize the appropriate reservoir model and pass the output choice to the parameter estimation algorithm which in turn calculates the permeability, skin factor, reservoir radius, flowing wellbore pressure and/or the length of the conductivity fracture.

\section{RESULT}

\subsection{Reservoir Model Recognition Results}

After the Reservoir Model Recognition training period, the following results were derived.

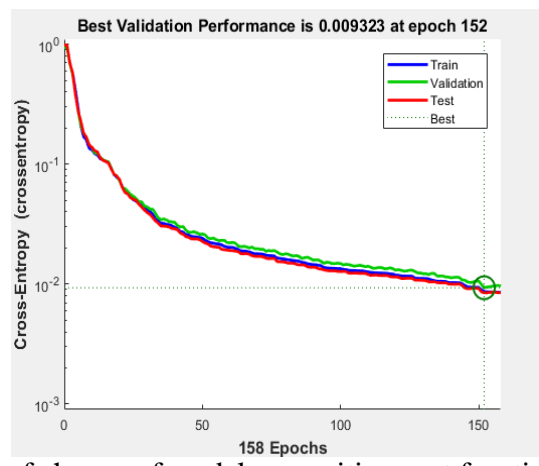

Figure 6: Plot of change of model recognition cost function to epoch (iteration) change

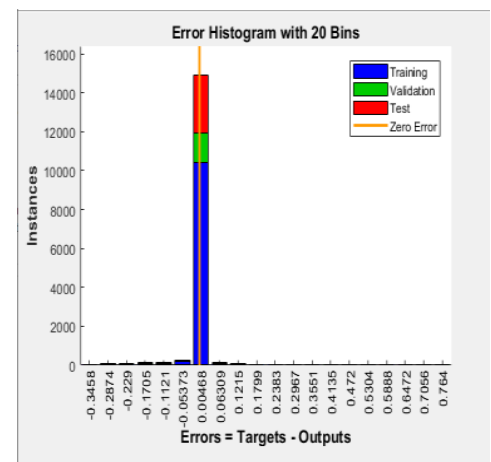

Figure 7: Reservoir model recognition error histogram

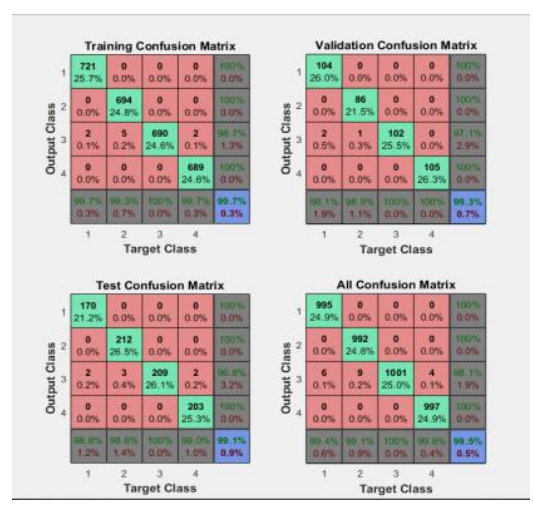

Figure 8: Reservoir model recognition confusion matrix

The model recognition algorithm took five minutes, thirtysix seconds to complete its training process. Figure 6 illustrates the performance (error function) of the algorithm and it indicates that after 152 epochs (iterations) the best performance was found to be 0.009323 . This point of best performances can be referred to as the point of global minimum or convergence point. Also, figure 7 describes the error histogram that shows the difference between the target and the output and its observed that the maximum instance lies at 0.00468. Also, figure 8 illustrates the confusion matrix of the algorithm. From the training confusion matrix, $25.7 \%$ (721) of the training data belongs to class 1 and the algorithm classified them correctly as class 1 while $0.1 \%$ (2) was misclassified as class 3 . On the other hand, $26.5 \%$ (694) of the training data belongs to class 2 and the algorithm classified them correctly as class 2 while the remaining $0.2 \%$ (5) was misclassified as class 3 also. Furthermore, $24.6 \%$ (690) of the training data belonging to class 3 was classified correctly as class 3 without any misclassification. In addition, 24.6\% (689) of the training data that belongs to class 4 was classified correctly as class 4 while $0.1 \%$ (2) was misclassified as class 3 . This gave the algorithm a training accuracy of $99.7 \%$. In other to make sure the algorithm generalizes and not over-fitted, the validation and testing are done. Testing is simply giving the algorithm data it has not seen before for it to classify. After the process of validation and testing, the validation accuracy was $99.3 \%$ and the test accuracy $99.1 \%$. This gave the algorithm a total of 99.5\% classification (Reservoir model recognition) accuracy.

\subsection{Reservoir Model Parameter Estimation Training Results}

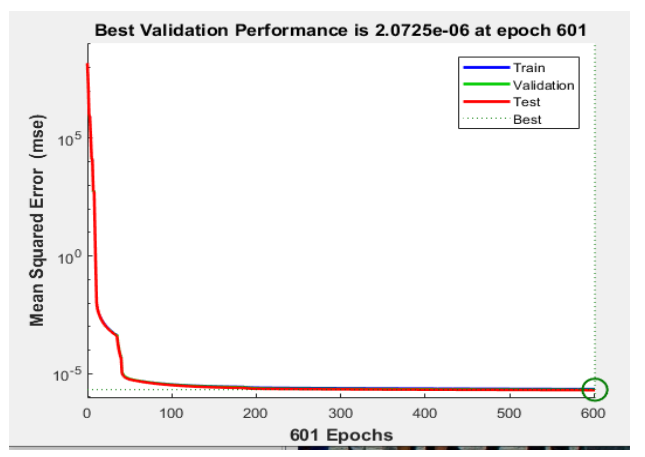

Figure 9: Mean square error change as number of iteration changes

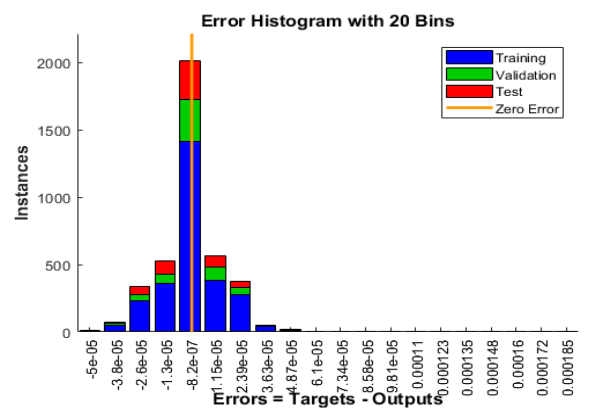


Figure 10: Reservoir model parameter estimation error histogram

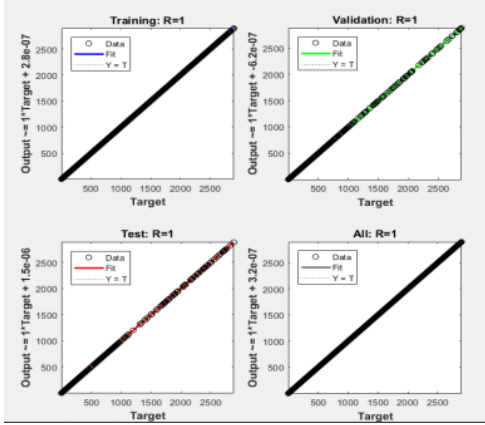

Figure 11: Regression plot covered in this work. The figures 9 to 11 above illustrates the results for class 1 only. After the training process, error and index of fitness for the different reservoirs are shown in table1 below;

The model parameter estimation algorithm was trained separately for the four different reservoir models that were

Table 1: Mean square error and index of fitness of the various reservoir algorithms

\begin{tabular}{|c|c|c|c|c|c|c|c|}
\hline \multirow[b]{2}{*}{$\begin{array}{l}\text { RESERVOIR } \\
\text { TYPE }\end{array}$} & \multirow{2}{*}{$\begin{array}{l}\text { TIME TO } \\
\text { TRAIN } \\
\text { (hr:min:sec) }\end{array}$} & \multicolumn{2}{|c|}{ TRAINING } & \multicolumn{2}{|c|}{ VALIDATION } & \multicolumn{2}{|l|}{ TESTING } \\
\hline & & $\begin{array}{l}\text { MSE } \\
\left(10^{-8}\right)\end{array}$ & $\mathbf{R}$ & $\begin{array}{l}\text { MSE } \\
\left(10^{-8}\right)\end{array}$ & $\mathbf{R}$ & $\begin{array}{l}\text { MSE } \\
\left(10^{-8}\right)\end{array}$ & $\mathbf{R}$ \\
\hline $\begin{array}{l}\text { Infinite acting } \\
\text { radial flow }\end{array}$ & $7: 12: 26$ & 0.0262653 & 1 & 0.0281048 & 1 & 0.0285931 & 1 \\
\hline $\begin{array}{l}\text { Finite } \\
\text { conductivity } \\
\text { fracture }\end{array}$ & $4: 34: 48$ & 3.29122 & 1 & 3.41237 & 1 & 3.34943 & 1 \\
\hline $\begin{array}{l}\text { infinite } \\
\text { conductivity } \\
\text { fracture }\end{array}$ & $5: 06: 18$ & 1058.05 & 1 & 1084.88 & 1 & 920.076 & 1 \\
\hline $\begin{array}{l}\text { Double porosity } \\
\text { reservoir }\end{array}$ & $3: 16: 12$ & 219.763 & 1 & 207.247 & 1 & 198.014 & 1 \\
\hline
\end{tabular}

Table 1 above shows that the error from the training, validation, and testing of the algorithms are minimal. Also, the index of fitness of 1 was recorded for all the reservoir types which indicate a perfect fit. Therefore, it is proper to infer that the algorithms have learnt to predict permeability, skin factor, reservoir extent, flowing bottom hole pressure and also the length of fracture of finite and infinite conductivity accurately. An analysis was made on a dataset from Horne (1990) example 6.1 shown in Figure 12 below.

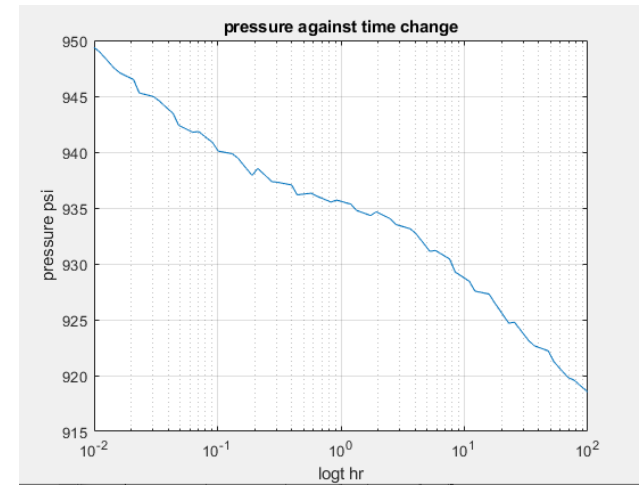

Figure 12: Well test data plot 


\section{International Journal of Engineering Applied Sciences and Technology, 2020 \\ Vol. 4, Issue 11, ISSN No. 2455-2143, Pages 438-446 \\ Published Online March 2020 in IJEAST (http://www.ijeast.com)}

This well-test dataset was passed into the algorithm and the result derived from this algorithm was compared with that of the conventional methods as solved by Horne (1990) and there was no significant error. Firstly, the algorithm detected the model to be a double porosity reservoir with a $100 \%$ certainty which is the same as Horne (1990) diagnosis. The estimated reservoir parameters are shown in table 2 below, it can be observed that the algorithm correctly identified the reservoir model and also predicted the reservoir parameters with a high degree of certainty and accuracy.

Table 2: Comparing study results with Horne (1990)

\begin{tabular}{|l|l|l|l|}
\hline PARAMETER & STUDY & $\begin{array}{l}\text { HORNE } \\
\mathbf{( 1 9 9 0 )}\end{array}$ & ERROR \\
\hline $\begin{array}{l}\text { Permeability } \\
\text { (md) }\end{array}$ & 498.6949 & 498.7 & -0.0051 \\
\hline Skin factor & -2.0082 & -2.007 & -0.0012 \\
\hline $\begin{array}{l}\text { Radius of } \\
\text { investigation } \\
\text { (ft) }\end{array}$ & 41280 & 41280 & 0.00 \\
\hline $\begin{array}{l}\text { Flowing bottom } \\
\text { hole pressure } \\
\text { (psi) }\end{array}$ & 918.4964 & 918.5 & -0.0036 \\
\hline
\end{tabular}

\section{CONCLUSION}

The objectives of a well test data analysis and interpretation as stated in section one above is to identify the conceptual reservoir model and estimate the identified reservoir model parameters. The algorithm proposed and developed in this paper was able to achieve the two objectives, although, constrained to only four reservoir types with a very high degree of certainty and accuracy. Therefore, it can be concluded that the artificial neural network algorithm is a good tool for well test analysis and interpretation and it saves both the time and energy of the interpreter when compared to conventional methods thereby providing enough time for proper engineering judgment.

Finally, in light of this, the following under listed are recommended for future consideration;

- In other to increase the capacity and accuracy of the algorithm, the algorithm should be retrained with more analyzed well-test data.

- Data should be made available for the other plethora of reservoir types in other to have a fully automated well test analysis and interpretation system.

- Verification and validation of the algorithm should be done with real-time data.
- Artificial intelligence should be welcomed and applied in the petroleum industry and also taught as a course in the petroleum engineering undergraduate program to harness its complete benefits.

\section{REFERENCE}

[1] Gringarten, A. C. "From Straight Lines to Deconvolution: The Evolution of the State of the Art in Well Test Analysis" SPE Reservoir Evaluation \& Engineering, (SPE 102079), pp. 41-62, 2008.

[2] Horne, R. N. "Advancement in Computer-Aided WellTest Interpretation". Journal of Petroleum Technology, (SPE 24731) pp. 599-606, 1994.

[3] Kumoluyi, A. O., Daltaban, T. S. and Archer, J. S. (1995) "Identification of Well-Test Models by Use of HigherOrder Neural Networks". SPE Computer Applications, (SPE 27558) pp. 146-150, 1995.

[4] Denney, D. "Spatial-Feature Identification and Parameter Estimation from Pressure-Transient Tests". Journal of Petroleum Technology, pp. 88-90, 2012.

[5] Spivey, J. P. and Lee, J. W. "Applied Well Test Interpretation". Richardson: SPE, 2013.

[6] Ahmadi, R., Shahrabi J. and Aminshahidy, B. "Automatic well-testing model diagnosis and parameter estimation using artificial neural networks and design of experiments". Journal of Petroleum Exploration and Production Technology, vol 7, pp. 750-783, 2016.

[7] Allain, O. F. and Home, R. N. "Use of Artificial Intelligence in Well Test Analysis," Journal of Petroleum Technology (March) pp. 342-349, 1990.

[8] Al-Kaabi, A. U. and Lee, W. J. "Using artificial neural networks to identify the well test interpretation model". Paper SPE20332 presented at the SPE petroleum conference, Denver, 1993.

[9] Ershaghi I., Li X., Hassibi M. and Shikari Y. "A Robust Neural Network Model for Pattern Recognition of Pressure Transient Test Data". Paper SPE26427 presented at the SPE annual technical conference and exhibition, Houston, Texas, USA, 1993.

[10] Aydinoglu, G., Bhat, M. and Ertkin, T. "Characterization of Partially Sealing Faults from Pressure Transient Data: An Artificial Neural Network Approach". Paper SPE78715 presented at the SPE eastern regional meeting, Lexington, Kentucky, USA, 2002.

[11] Deng, Y., Chen, Q., and Wang, J. 'The Artificial Neural Network Method of Well-Test interpretation Model Identification and Parameter Estimation'. Paper SPE 64652 presented at the SPE international Oil and Gas Conference and Exhibition held in Beijing, China, 7-10 November, 2000. 


\section{International Journal of Engineering Applied Sciences and Technology, 2020 \\ Vol. 4, Issue 11, ISSN No. 2455-2143, Pages 438-446 \\ Published Online March 2020 in IJEAST (http://www.ijeast.com)}

[12] Bourdet, D., Whittle, T.M., Douglas, A.A., Pirard, Y.M. and Kniazeff, V. J. "Interpreting Well Tests in Fractured Reservoirs," World Oil pp. 95-106, October, 1983

[13] Gringarten A. C. "Computer Aided Well Test Analysis". Paper SPE14099 presented at the SPE International Meeting on Petroleum Exploration held 17-20 March in Beijing, China, 1986.

[14] AI-Kaabi A.U., McVay, D.A. and Lee W.J. "Using an Expert System to Identify a Well Test Interpretation Model". Journal of Petroleum Technology, (May), pp. 654-661, 1990.

[15] Allain, O.F. and Houze, O. P. "A Practical Artificial Intelligence Application in Well Test Interpretation". Paper SPE 24287 presented at the SPE European Petroleum Computer Conference, held in Stavenger, Norway, 25-27 May, 1992.

[16] Sinha, S., and Panda, M. N. "Well-Test Model Identification with Self-Organizing Feature Map". SPE Computer Application (August) SPE 30216, 1996.

[17] Sung, W., Hanyang, U., and Yoo, I. "Development of HT-BP Neural Network System for identification of well Test Interpretation Model'. Paper SPE 30974 presented at the Eastern Regional Meeting, held in Morgan-town, West Virginia, 17-21 September, 1995.

[18] Lu, P., and Horne, R. N. "Multiresolution Approach to Reservoir Parameter Estimation Using Wavelet Analysis". Paper SPE 62985 presented at the SPE Annual Technical Conference and Exhibition, held in Dallas, USA, 1-4 October, 2000.

[19] Davidson, W.C. "Variable Metric Method for Minimization". Tech Report, ANL-5590, Argonne National Lab, Illinois, 1959.

[20] Levenberg, K. "A Method for the Solution of Certain Non-linear Problems in Least Squares". The Quarterly of Applied Mathematics vol 2 pp. 164-168,1944.

[21] Marquardt, D. "An Algorithm for Least Square Estimation of Nonlinear Parameters". SIAM journal of Applied Mathematics vol. 11 pp. 431-441, 1963.

[22] Fletcher, R. and Powell, M.J.D. "A Rapidly Convergent Descent Method for Minimization”. Computer Journal, vol 6, pp. 163-168, 1963.

[23] Nashed, M.D. "Steepest Descent for Singular Linear Operator Equations". SIAM journal of Numeric Analysis, vol 7(3), pp. 358-362, 1970.

[24] Fletcher, R. and Reeves, C.M. "Function Minimization by Conjugate Gradient' Computer Journal vol 7, pp. 149154, 1964.
[25] Gill, P.E. Murray, W. and Wright, M.H. "Practical Optimization". New York: Academic Press, 1981.

[26] Grivia, I., Nash, S.G, and Sofer, A. "Linear and Nonlinear Optimization". Philadelphia, PA: SIAM Press, 2009.

[27] Padmanabhan, L. and Woo, P.T. "A New Approach to Parameter Estimation in Well Testing”. Paper SPE 5741 presented at the Fourth Symposium of Numerical Simulation of Reservoir Performance of the Society of Petroleum Engineers of AIME held in Los Angeles, California, 1976.

[28] Wilkinson, D.J. "Pressure Transient Parameter Estimation in the Laplace Domain". Paper SPE24720 presented at SPE meetings, 1992.

[29] Bonalde, I., and Ramones, M. "A Robust Algorithm for Parameter Estimation in Well Tests". SPE advanced Technology series, vol 2(1), SPE23656, 1994.

[30] Sung, W., Hanyang, U., and Yoo, I. "Development of HT-BP Neural Network System for identification of well Test Interpretation Model'. Paper SPE30974 presented at the Eastern Regional Meeting, held in Morgan-town, West Virginia, 17-21 September, 1995.

[31] Athichanagorn, S. and Horne, R.N. "Automatic Parameter Estimation From Well Test Data Using Artificial Neural Network". Paper SPE30556 presented at the SPE Annual Technical Conference and Exhibition held in Dallas, USA, 22-25 October, 1995.

[32] Martinez-Romero, N. Samaniego, F.V., Cinco-Ley, H. and Villagran J.A. "Automated Parameter Estimation from Well Test Data Using the Signal Theory". Paper SPE92240 presented at the SPE International Petroleum Conference held in Puebla, Mexico, 8-9 November, 2004.

[33] Biu, V.T. (2010) 'Well and Reservoir Parameters Estimation (K and Skin) Using the Statistical Diagnostic Approach Part II' Paper SPE 136963 presented at the 34th Annual SPE Internation Conference and Exhibition held in Tinapa, Calabar, Nigeria, 31 July - 7 August, 2010.

[34] Morton, K.L., Booth, R.J., Onur, M. and Kuchuk, F.J. "Grid-Based Inversion Methods for Spatial Feature Identification and Parameter Estimation from Pressure Transient Tests". Paper SPE 142996 presented at the SPE EUROPEC/EAGE Annual Conference and Exhibition held in Vienna, Austria, 23-26 May, 2011.

[35] Awotunde, A.A. (2012) 'Reservoir Parameter Estimation with Improved Particle Swarm Optimization'. Paper SPE 159470 presented at the SPE Annual Technical Conference and Exhibition held in San Antonio, Texas, USA, 8-10 October, 2012. 